\title{
Computerized Dead-Space Volume Measurement of Face Masks Applied to Simulated Faces
}

\author{
Israel Amirav MD, Anthony S Luder MD, Asaf Halamish MSc, Chatib Marzuk, \\ Marcelo Daitzchman MD, and Michael T Newhouse MD MSc FRCP(C)
}

\begin{abstract}
BACKGROUND: The dead-space volume $\left(V_{D}\right)$ of face masks for metered-dose inhaler treatments is particularly important in infants and young children with asthma, who have relatively low tidal volumes. Data about $V_{D}$ have been traditionally obtained from water displacement measurements, in which masks are held against a flat surface. Because, in real life, masks are placed against the face, $V_{D}$ is likely to differ considerably between masks depending upon their contour and fit. The aim of this study was to develop an accurate and reliable way to measure $V_{D}$ electronically and to apply this technique by comparing the electronic $V_{D}$ of commonly available face masks. METHODS: Average digital faces were obtained from 3-dimensional images of 270 infants and children. Commonly used face masks (small and medium) from various manufacturers (Monaghan Medical, Pari Respiratory Equipment, Philips Respironics, and InspiRx) were scanned and digitized by means of computed tomography. Each mask was electronically applied to its respective digital face, and the $V_{D}$ enclosed $(\mathrm{mL})$ was computerized and precisely measured. RESULTS: $V_{D}$ varied between $22.6 \mathrm{~mL}$ (SootherMask, InspiRx) and $43.1 \mathrm{~mL}$ (Vortex, Pari) for small masks and between $41.7 \mathrm{~mL}$ (SootherMask) and $71.5 \mathrm{~mL}$ (AeroChamber, Monaghan Medical) for medium masks. These values were significantly lower and less variable than measurements obtained by water displacement. CONCLUSIONS: Computerized techniques provide an innovative and relatively simple way of accurately measuring the $V_{D}$ of face masks applied to digital faces. As determined by computerized measurement using average-size virtual faces, the InspiRx masks had a significantly smaller $V_{D}$ for both small and medium masks compared with the other masks. This is of considerable importance with respect to aerosol dose and delivery time, particularly in young children. (Clinical Trials.gov registration NCT01274299.) Key words: masks; dead space; aerosol; infants. [Respir Care 2015;60(9):1247-1251. (c) 2015 Daedalus Enterprises]
\end{abstract}

\section{Introduction}

Aerosol delivery to infants and young children with asthma has advanced greatly since the introduction of

\footnotetext{
Drs Amirav, Luder, and Daitzchman and Mr Marzuk are affiliated with the Ziv Medical Center, Faculty of Medicine, Bar-Ilan University, Safed, Israel. Mr Halamish is affiliated with Technosaf, Karkur, Israel. Dr Newhouse is affiliated with the Firestone Institute for Respiratory Health, St Joseph's Hospital, McMaster University, Hamilton, Ontario, Canada.
}

Dr Newhouse has disclosed a relationship with InspiRx. Drs Amirav and Newhouse have patent rights for devices that deliver aerosols to infants. The other authors have disclosed no conflicts of interest.

Correspondence: Israel Amirav MD, Department of Pediatrics, University of Alberta, 116 Street and 85 Avenue, Edmonton, Alberta T6G 2R3, Canada. E-mail: amirav@012.net.il.

DOI: $10.4187 /$ respcare. 03813 valved holding chambers a few decades ago. During this time, there have been many important developments in valved holding chamber design. Because infants and young children under $5 \mathrm{y}$ of age cannot reliably use a mouthpiece, they must use a face mask. However, as the tidal volume $\left(\mathrm{V}_{\mathrm{T}}\right)$ of these infants is relatively low, there should be the smallest possible dead space between the inspiratory and expiratory ports of the mask to optimize aerosol delivery. The dead-space volume $\left(\mathrm{V}_{\mathrm{D}}\right)$ of a face mask has been shown to critically affect aerosol delivery to infants and young children. ${ }^{1,2}$

Traditionally, data about $\mathrm{V}_{\mathrm{D}}$ have been obtained from water displacement measurements when masks are held against a flat surface, ${ }^{1,3}$ with the inlet and outlet valves occluded. Because, in real life, masks are placed against the face, this functional $V_{D}$ is not obtained using the water displacement technique, which measures only the total volume of the mask and not $\mathrm{V}_{\mathrm{D}}$. Furthermore, $\mathrm{V}_{\mathrm{D}}$ may differ 
considerably if mask contour, fit, and applied pressure vary. ${ }^{2}$ Indeed, there are many available face masks that differ not only in their $\mathrm{V}_{\mathrm{D}}$, but also in their ability to align with the facial contour of the patient. The purpose of this study was to develop an accurate and reliable way to measure electronic $\mathrm{V}_{\mathrm{D}}$ and then apply this technique to a comparison of the electronic $V_{D}$ of commonly available face masks.

\section{Methods}

Two average digital faces (small and medium) were obtained from 3-dimensional images of infants and children included in a previous study. ${ }^{4}$ In brief, faces of 271 children (0-4 y of age) were scanned with 3-dimensional structured light technology, which converts the facial surface into a triangulated mesh-like surface. Ethical approval and parental informed consent for scanning the children's faces were mentioned in the earlier publication outlining this methodology. ${ }^{4}$ These 3 -dimensional images were analyzed using pattern recognition software (TechnionIsrael Institute of Technology, Haifa, Israel). The vertical dimension from the nadir of the nasal bridge to the apex of the chin was found to be the best measure of mask size. The data were used to categorize the facial scans into small, medium, and large clusters. There was no effect of age or sex on these measures. Average digital face models were developed for each cluster and were kept as electronic files in the computer. Detailed description of the averaging methods is described elsewhere. ${ }^{4}$ Face masks were scanned by computed tomography (Brilliance CT 64-channel scanner, Philips Healthcare, Best, The Netherlands), and the data were digitized and converted to electronic files. Technical parameters included: $64 \times 0.625$ collimation, 0.75 -s pitch, $120 \mathrm{kV}, 100 \mathrm{~mA}$, and a 2 -s rotation time. The thickness of a slice was $2.5 \mathrm{~mm}$, with increments of $1.25 \mathrm{~mm}$. Slices were stored in DICOM (Digital Imaging and Communications in Medicine) format. Subsequently, the slices were joined together to compose a 3-dimensional reconstruction image, which was stored in STL (Standard Transformation Language) format, and later transferred over local area network (LAN) for further analysis.

Because we were particularly interested in the younger age groups, we focused on the small and medium masks provided by various manufacturers. Using a specially designed software engine (SolidWorks, Waltham, Massachusetts), we transformed each STL file into a computer-aided design (CAD) file. The mask and face CAD files were fixed geometric shapes that were not deformable and were not influenced by the operator. Using the same software, the digital small electronic masks were aligned with the digital small average face, and the digital medium

\section{QUICK LOOK}

\section{Current knowledge}

Dead-space volume $\left(\mathrm{V}_{\mathrm{D}}\right)$ of face masks for metereddose inhaler treatments is particularly important in infants and young children, who have relatively low tidal volumes, and in those with decreased compliance causing shallow breathing due to disease. Previous methods to measure $V_{D}$ were based on water displacement of masks.

\section{What this paper contributes to our knowledge}

Computerized techniques were used to provide an innovative way to accurately measure the $V_{D}$ of face masks applied to virtual faces obtained from images of real infants and children. The technique allows for a virtual in situ comparison of various face masks.

electronic masks were aligned with the digital medium average face. The rims of the masks were attached electronically to the face with the aim of achieving the best seal and fit (Fig. 1). To verify the method, each application of each electronic mask was repeated 3 times. The electronic $\mathrm{V}_{\mathrm{D}}(\mathrm{mL})$ between the digital face and the interior of the electronic mask was then computed. Three measurements were carried out for each mask under each condition. The masks were aligned as is, with no attempt to simulate any application forces that could have changed the static volume of the masks.

To further compare the new technique with the traditional water displacement technique, the same masks were held upside down, and ports were occluded and filled with water to a leveled surface. The volume of water was measured using volume-calibrated syringes. ${ }^{3,5}$ The mean of 3 determinations was recorded. Student $t$ tests were used to assess differences between techniques (SPSS Statistics 22, IBM, Armonk, New York).

We compared commercially available small and medium masks: AeroChamber Plus Flow-Vu with ComfortSeal mask (Monaghan Medical, Plattsburgh, New York), SmartTouch pediatric Vortex mask (Pari Respiratory Equipment, Midlothian, Virginia), OptiChamber Diamond LiteTouch mask (Philips Respironics, Murrysville, Pennsylvania), and SootherMask and InspiraMask (InspiRxpharma Inc, Durham, North Carolina) (Fig. 2).

\section{Results}

The electronic $V_{D}$ values for both small and medium masks are shown in Tables 1 and Table 2, respectively. The range of electronic $V_{D}$ was from $22.6 \mathrm{~mL}$ (Soother- 

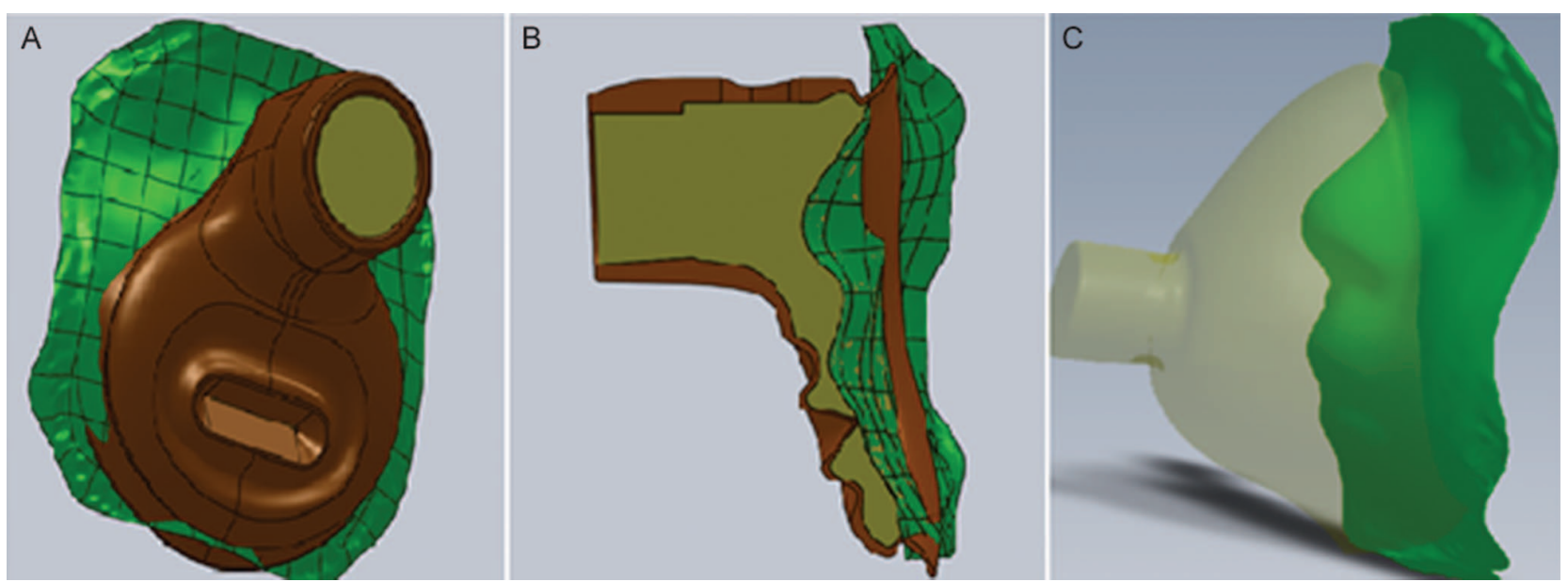

Fig. 1. Electronic application of computed tomography using scanned and digitized face masks and face models. A: SootherMask, anterior view. B: SootherMask, lateral view. C: Non-contoured mask, lateral view.

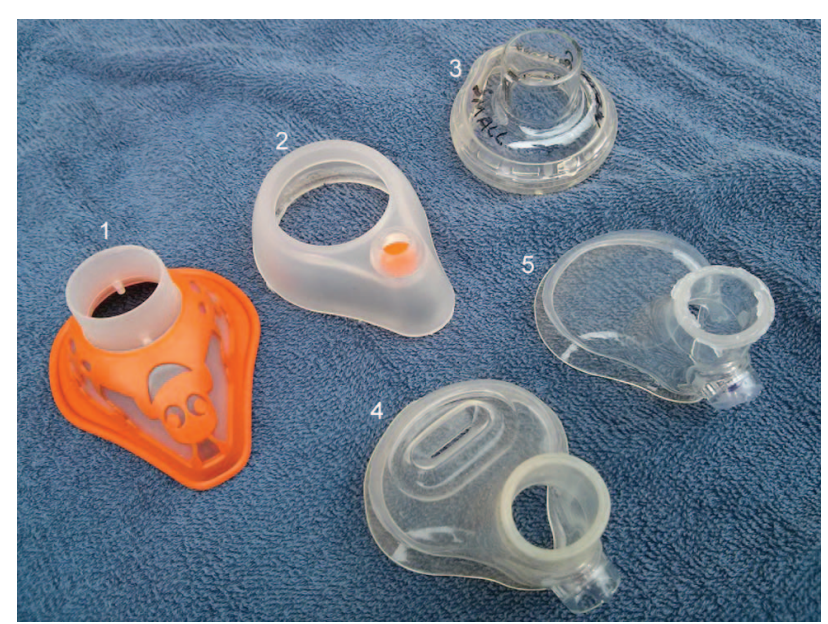

Fig. 2. Masks used in the study (small sizes shown). 1: Vortex. 2: AeroChamber. 3: OptiChamber. 4: SootherMask. 5: InspiraMask.

Mask) to $43.1 \mathrm{~mL}$ (Vortex) for the small masks and from $41.7 \mathrm{~mL}$ (SootherMask) to $71.5 \mathrm{~mL}$ (AeroChamber) for the medium masks. Measurements of the same mask using the water displacement technique were significantly higher $(P<.01)$ than those using the electronic technique. The variance was also significantly higher $(P<.001)$ for the water displacement measurement (mean of $2.3 \%$ ) compared with the electronic $\mathrm{V}_{\mathrm{D}}$ (mean of $0.5 \%$ ).

\section{Discussion}

Face masks used for aerosol delivery to infants and small children with asthma constitute one of the most important links in the chain from aerosol generator to the respiratory tract of a child. With regard to the efficiency of aerosol delivery to the lungs, particularly in infants and toddlers, one of the most important factors is the volume of air common to the inspiratory and expiratory pathways, namely, the mask $\mathrm{V}_{\mathrm{D}}$. With valved holding chambers, any residual drug from an aerosol reservoir, such as the InspiraChamber (InspiRx), contained in that volume will be lost on expiration and thus will not contribute to the lung dose. Smaller mask $V_{D}$ values result in a greater proportion of the dose in the valved holding chamber being inhaled with each breath, ${ }^{6}$ thus accelerating chamber emptying and improving overall aerosol delivery efficiency and lower respiratory tract dose. Chavez et al $^{1}$ studied albuterol delivery via an AeroChamber and showed that greater dead space of a mask attached to this chamber resulted in less efficient aerosol delivery for the same $\mathrm{V}_{\mathrm{T}}{ }^{1}$ Thus, the $\mathrm{V}_{\mathrm{D}}$ of a face mask is a very important determinant of face-mask design, ${ }^{3}$ particularly in infants and small children with low $\mathrm{V}_{\mathrm{T}}$. The mean $\mathrm{V}_{\mathrm{T}}$ in infants 9 months old was recently documented as $81.5 \pm 28.2 \mathrm{~mL}$, with a mean $\mathrm{V}_{\mathrm{T}}$ of $9.7 \pm 1.3 \mathrm{~mL} / \mathrm{kg} .{ }^{7}$ Additionally, the $\mathrm{V}_{\mathrm{T}}$ may be variably reduced in a variety of diseases (eg, pneumonia, cystic fibrosis, bronchopulmonary dysplasia) characterized by rapid breathing frequency and stiff, low-compliance lungs.

This work describes a new method to evaluate the dead space of face masks. The method entails scanning face masks by computed tomography, digitizing the data, and electronically applying the mask to appropriate size- and age-specific electronic faces. Computerized objective measurements can then be employed to derive the actual dead space of the mask when applied to the electronic face.

Previous studies used water displacement to measure dead space. ${ }^{1,8}$ This technique has limited value, as it usually requires that the mask be placed against a smooth, flat surface. However, children's faces are far from flat, and 


\section{Face-Mask Dead Space}

Table 1. Mean and Variance of $\mathrm{V}_{\mathrm{D}}$ for Small Masks Using Electronic and Water Displacement Techniques

\begin{tabular}{|c|c|c|c|c|c|}
\hline Technique & AeroChamber & Vortex & OptiChamber & InspiraMask & SootherMask \\
\hline Electronic $\mathrm{V}_{\mathrm{D}}$, mean $\pm \mathrm{SD} \mathrm{mL}$ & $39.6 \pm 0.2$ & $43.1 \pm 0.2$ & $36.9 \pm 0.1$ & $25.8 \pm 0.1$ & $22.6 \pm 0.2$ \\
\hline Coefficient of variation, $\%$ & 0.5 & 0.5 & 0.3 & 0.4 & 0.9 \\
\hline Water displacement, mean $\pm \mathrm{SD} \mathrm{mL}$ & $49.0 \pm 0.02$ & $45.3 \pm 0.03$ & $49.0 \pm 0.02$ & $27.6 \pm 0.02$ & $26.3 \pm 0.02$ \\
\hline Coefficient of variation, $\%$ & 2.0 & 3.4 & 2.0 & 2.1 & 2.2 \\
\hline
\end{tabular}

Table 2. Mean and Variance of $\mathrm{V}_{\mathrm{D}}$ for Medium Masks Using Electronic and Water Displacement Techniques

\begin{tabular}{|c|c|c|c|c|c|}
\hline Technique & AeroChamber & Vortex & OptiChamber & InspiraMask & SootherMask \\
\hline Electronic $\mathrm{V}_{\mathrm{D}}$, mean $\pm \mathrm{SD} \mathrm{mL}$ & $71.5 \pm 0.3$ & $49.2 \pm 0.3$ & $51.7 \pm 0.3$ & $42.3 \pm 0.2$ & $41.7 \pm 0.2$ \\
\hline Coefficient of variation, $\%$ & 0.4 & 0.6 & 0.6 & 0.5 & 0.5 \\
\hline Water displacement, mean $\pm \mathrm{SD} \mathrm{mL}$ & $90.3 \pm 0.02$ & $69.6 \pm 0.03$ & $108.3 \pm 0.01$ & $51.6 \pm 0.03$ & $51.6 \pm 0.03$ \\
\hline Coefficient of variation, $\%$ & 1.7 & 3.0 & 1.4 & 3.0 & 3.0 \\
\hline
\end{tabular}

moreover, they develop and grow continuously and relatively rapidly. Thus, the mask needs to be adapted to both the rapidly changing size and contours of the face associated with a continuously changing dead space. Only a few studies have tried to use water displacement of masks aligned with a model face rather than with a flat surface. Morris ${ }^{5}$ suggested a more sophisticated technique for water displacement measurements; however, it applied to sedated infants. Water displacement was later used by Shah et $\mathrm{al}^{2}$ to measure the $\mathrm{V}_{\mathrm{D}}$ of masks attached to a 2 -y-old mannequin's face, whereas Mundt et $\mathrm{al}^{9}$ used it to measure the $\mathrm{V}_{\mathrm{D}}$ of a Pari mask applied to a face and upper airway model. In comparison with the traditional water displacement technique, the electronic $V_{D}$ values obtained in the present study were significantly smaller, as they more accurately reflect the actual dead space of the masks aligned with the face rather than with a flat surface. Moreover, the variability of the new technique is significantly smaller compared with the traditional water displacement technique.

Some limitations of our study should be acknowledged. The method described here measures only the static volume of a mask. Because the $V_{D}$ may change with varying application forces and flexible materials, ${ }^{2,3}$ these factors may be relevant in future studies of pediatric mask design. It may be of interest to modify the software to simulate these conditions as well. We have not measured drug delivery in this study, and it is known that in addition to $\mathrm{V}_{\mathrm{D}}$, several other factors may affect drug delivery. ${ }^{10}$

\section{Conclusions}

In summary, computerized technology allows for more accurate spatial measurements. The faces recorded from a large number of infants were obtained with no difficulty and with no need for sedation or invasive techniques. Computerized evaluation of electronic files facilitated precise evaluation and comparison of the $V_{D}$ of commercially available face masks commonly used for aerosol delivery. The masks with the smallest electronic $V_{D}$ were the InspiRx SootherMask and InspiraMask, which were designed to minimize dead space. We suggest that this computerized technique would be a useful approach when new masks are being developed.

\section{REFERENCES}

1. Chavez A, McCracken A, Berlinski A. Effect of face mask dead volume, respiratory rate, and tidal volume on inhaled albuterol delivery. Pediatr Pulmonol 2010;45(3):224-229.

2. Shah SA, Berlinski AB, Rubin BK. Force-dependent static dead space of face masks used with holding chambers. Respir Care 2006; 51(2):140-144.

3. Amirav I, Newhouse MT. Dead space variability of face masks for valved holding chambers. Isr Med Assoc J 2008;10(3):224-226.

4. Amirav I, Luder AS, Halamish A, Raviv D, Kimmel R, Waisman D, Newhouse MT. Design of aerosol face masks for children using computerized 3D face analysis. J Aerosol Med Pulm Drug Deliv. 2014;27(4):272-278.

5. Morris MG. A simple new technique to measure the effective dead space of the face mask with a water volumeter in infants. Eur Respir J 1999;14(5):1163-1166. 


\section{Face-Mask Dead Space}

6. Turpeinen M, Nikander K, Malmberg LP, Pelkonen A. Metered dose inhaler add-on devices: is the inhaled mass of drug dependent on the size of the infant? J Aerosol Med 1999;12(3):171-176.

7. Nguyen TT, Hoo AF, Lum S, Wade A, Thia LP, Stocks J. New reference equations to improve interpretation of infant lung function. Pediatr Pulmonol 2013;48(4):370-380.

8. Amirav I, Newhouse MT. Aerosol therapy with valved holding chambers in young children: importance of facemask seal. Pediatrics 2001; 108(2):389-394
9. Mundt C, Sventitskiy A, Cehelsky JE, Patters AB, Tservistas M, Hahn MC, et al. Assessing modeled $\mathrm{CO}_{2}$ retention and rebreathing of a facemask designed for efficient delivery of aerosols to infants. ISRN Pediatr 2012;2012:721295.

10. Carrigy NB, O'Reilly C, Schmitt J, Noga M, Finlay WH. Effect of facial material softness and applied force on face mask dead volume, face mask seal, and inhaled corticosteroid delivery through an idealized infant replica. J Aerosol Med Pulm Drug Deliv 2014;27(4):290-298.

This article is approved for Continuing Respiratory Care Education credit. For information and to obtain your CRCE

(free to AARC members) visit

www.rcjournal.com 